\title{
Molecular cloning and functional characterization of chalcone isomerase from Carthamus tinctorius
}

\author{
Xiuming Liu ${ }^{1,2^{*}}$, Naveed Ahmad ${ }^{1,2}$, Longyu Yang ${ }^{2}$, Tianyu Fu², Jie Kong ${ }^{2}$, Na Yao ${ }^{1}$, Yuanyuan Dong ${ }^{1}$, Nan Wang ${ }^{1}$, \\ Xiaowei Li ${ }^{1}$, Fawei Wang ${ }^{1}$, Xin Liu' ${ }^{1}$, Weican Liu ${ }^{1}$ and Haiyan Li ${ }^{1}, 2^{*}$
}

\begin{abstract}
Flavonoid is one of the widespread groups of plant secondary metabolites that provide several health benefits. However, the explicit mechanism of flavonoid biosynthesis in plants largely remains unclear. Chalcone isomerase an important class of enzyme presents crucial role during flavonoid metabolism in many plants. Here, we isolated the full-length cDNA (1161 bp) of a novel Chalcone Isomerase from safflower encoding 217 amino acid polypeptide using oligos from $5^{\prime}$ and $3^{\prime}$ ends. The result of Sanger sequencing and phylogenetic analysis revealed that $\mathrm{CtCH}$ is highly homologous to other plants, including typical polyadenylation signals AATAA and Poly A tail. The transient expression in tobacco mesophyll cells using Green Fluorescent Protein tagging determined the subcellular localization of $\mathrm{CtCHI}$ in cell membrane and nucleus. The $\mathrm{CtCH}$ ectopic expression in different safflower varieties at different flowering stages showed that $\mathrm{CtCH}$ I were found in abundance at the bud stage of Jihong No. 1. Further correlation analysis between $\mathrm{CtCH}$ expression and flavonoid accumulation at various flowering phases suggested that $\mathrm{CtCH}$ might play a potential role during flavonoid biosynthesis in safflower. In addition, the overexpression of pBASTA-CtCHI in transgenic Arabidopsis infiltrated with floral dip transformation showed relatively higher expression level and increased flavonoid accumulation than wild type. Moreover, the in vitro enzymatic activity and HPLC analysis of transgenic Arabidopsis confirmed the de novo biosynthesis of Rutin. Taken together, our findings laid the foundation of identifying an important gene that might influence flavonoid metabolism in safflower.
\end{abstract}

Keywords: Chalcone isomerase (CHI), Flavonoids biosynthesis, Safflower, Expression analysis, HPLC

\section{Introduction}

Safflower (Carthamus tinctorius L. Compositae), a dicotyledonous plant, serves as an important traditional Chinese herb. It is not only used for medicinal purposes, but also as a food supplement in various kinds of nutritional oil. Safflower has been valued historically for its abundant Flavonoids content, fatty acids, various phenolic compounds, and lignin product (Dai et al. 2013). Flavonoids are one of the most important phenolic compounds (Guo et al. 2016; Yaginuma et al. 2003). Flavonoids are

\footnotetext{
*Correspondence: xiuming1211@163.com; hyli99@163.com

${ }^{1}$ Ministry of Education Engineering Research Center of Bioreactor and Pharmaceutical Development, Jilin Agricultural University,

Changchun 130118, China

Full list of author information is available at the end of the article
}

widely distributed class of plant producing secondary metabolites linked with a variety of metabolic functions in plants. Among secondary metabolites, the biosynthesis pathway used to produce flavonoid compounds is one of the most thoroughly elucidated metabolic pathways. In Arabidopsis, flavonoids are synthesized naturally by catalyzing $p$-coumaroyl-CoA and 3 malonyl-CoA molecules with the help of chalcone synthase $(\mathrm{CHS})$ which is prearranged on TT4 locus. The first product in this stepwise condensation manner is naringenin chalcone, which is then undergoing isomerization via chalcone isomerase $(\mathrm{CHI})$ to form naringenin (Fig. 1). The second product (naringenin) is considered the basic intermediate of the flavonoid pathway. Following other metabolic routes during flavonoid metabolism naringenin is further converted to dihydrokaempferol with the help of flavanone 
<smiles>CC(C(=O)O)c1c(O)cc(O)c(C(=O)/C=C/c2ccc(O)cc2)c1O</smiles>

Naringenin chalcone<smiles>O=C1CC(c2ccc(O)cc2)Oc2cc(O)cc(O)c21</smiles>

Naringenin<smiles>O=C1c2c(O)cc(O)cc2OC(c2ccc(O)cc2)C1O</smiles>

Dihydrokaempferol

Dihydroquercetin

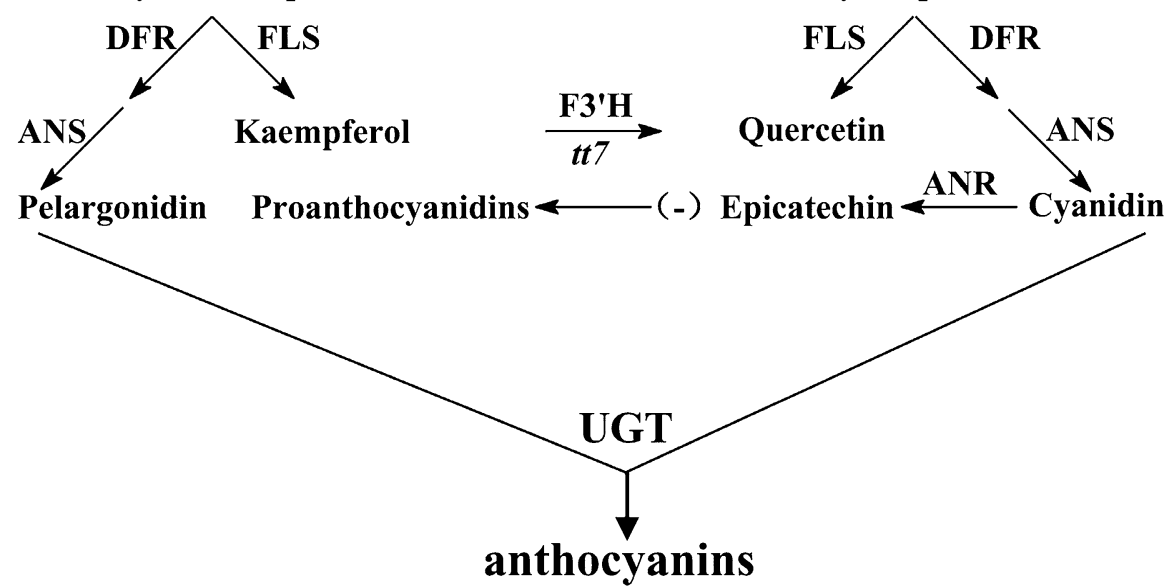

Fig. 1 The core metabolic pathway of flavonoids (anthocyanins) occurred in Arabidopsis thaliana (Jiang et al. 2015)

3-hydroxylase enzyme $(F 3 H)$ encoded by TT6 locus. Further stepwise hydroxylation of dihydrokaempferol can lead to the formation of dihydroquercetin by flavanone $3^{\prime}$-hydroxylase $\left(F 3^{\prime} H\right)$. The aforementioned two important intermediates can be converted to other distinct groups of flavonoids such as anthocyanins, and flavonols 
and phenylalanines with the help of a complex group of multienzymes including anthocyanidin synthase (ANS), flavonol synthase $(F L S)$, dihydroflavonol 4-reductase $(D F R)$, UDP-glucosyltransferase (UGT) and anthocyanidin reductase (ANR) (Jiang et al. 2015; Meng et al. 2015).

Much effort has been given to elucidate flavonoid biosynthetic pathways from a genetic perspective. To date, most of the structural and several regulatory genes involved in the flavonoid biosynthesis pathway have been cloned in a variety of plants (Li 2014; Tu et al. 2016; Wang et al. 2016). The most important genes or their antisense sequences in the secondary metabolism of flavonoids have been transferred into other plant species through genetic engineering, leading to changes in the synthesis of flavonoid compounds by promoting or inhibiting the expression of these genes (Gutierrez et al. 2017). However, the biosynthesis pathway of flavonoids involves a multienzyme complex system including isomerases, reductases, hydroxylases, and several $\mathrm{Fe}^{2+} / 2$-oxoglutarate-dependent dioxygenases, depending on the species involved. In addition, flavonoids play an important role in the plant response to adverse environments in vivo, for example, they can work as scavengers of free radicals such as reactive oxygen species (ROS) or being involved in the resistance of plants to drought (Jamalan et al. 2016; Kranner and Birtić 2005; Landry et al. 1995).

Recently, a number of CHI genes have been extensively characterized from different plant species (Dastmalchi and Dhaubhadel 2015; Jiang et al. 2015; Kang et al. 2014) and other functional genes were also identified during natural flavonoid biosynthetic pathway in Carthamus tinctorius including CHS (Guo et al. 2017), UGTS (Guo et al. 2016). However, there is no such report available on $\mathrm{CHI}$ from Carthamus tinctorius and is unreported up to date. Chalcone isomerase (CHI, EC:5.5.1.6), was found a key enzyme during flavonoid metabolic pathway in several other plants (Gensheimer and Mushegian 2004) which catalyze the isomerization of chalcones into their corresponding (-)-flavanones. $\mathrm{CHI}$ is commonly found in the form of monomers in most plant species with remarkably variable molecular weights. Genes of the $\mathrm{CHI}$ family generally encode proteins with $210-240$ amino acids with a high frequency of conserved sequences. Moreover, the sequence homologies among different species are reported between 50 and $80 \%$. Here, we presented the first report on the discovery and characterization of a new chalcone isomerase gene from safflower cultivar Jihong No. 1 using an expressed sequence homology-based approach followed by subcellular localization in tobacco mesophyll cells through GFP tagging. In addition, the expression analysis in the transgenic Arabidopsis T3 homozygous plants was also carried out using real-time quantitative PCR analysis. For this purpose, the overexpressed lines of Arabidopsis thaliana harboring (PBASTA-CtCHI) construct were generated through floral dip transformation. Homozygous transgenic T3 plants were selected for further characterization, including expression analysis, in vitro enzymatic activity and HPLC analysis. Our findings implied the discovery of a novel chalcone isomerase gene which could induce the flavonoid biosynthetic pathway in Carthamus tinctorius.

\section{Materials and methods}

Strains, cells and experimental materials

Safflower seeds were provided by Fuyu seeds company, China. Escherichia coli BL21 (DE3), Escherichia coli TransT1, DH5 $\alpha$ competent cells, and A. tumefacien strain EHA105 were purchased from Takara Biotechnology Company Beijing, China. Plant overexpression vector (pBASTA) and pCAMBIA1302-GFP-35S were purchased from TransGen.

Biotech, Beijing and preserved in our laboratory (Ministry of Education Engineering Research Center of Bioreactor and Pharmaceutical Development at Jilin Agricultural University, Jilin, China) until next use. Restriction enzymes, $\mathrm{G}^{\circ} \mathrm{Taq}$ DNA polymerase and DNA ligases were purchased from Takara Biotechnology Company Beijing, China.

\section{Plant materials}

Four safflower cultivars, Jihong (early maturing line) (JHEM), Jihongyou sister line (JHS), Jihong No. 1 (JH1), and Jihong No. 2 (JH2) were grown at the experimental station of the Ministry of Education Engineering Research Center of Bioreactor and Pharmaceutical Development at Jilin Agricultural University, Jilin, China. Flower petals of each cultivar at the bud, initial, full, and fade stages were sampled and wrapped with tin foil, quickly placed into liquid nitrogen, and then stored at $-80{ }^{\circ} \mathrm{C}$. Flower petals of each cultivar at the bud, initial, full, and fade stages were sampled and wrapped with tin foil, quickly placed into liquid nitrogen, and then stored at $-80{ }^{\circ} \mathrm{C}$. Wild-type (WT) A. thaliana was grown for 5-6 weeks, and flowering plants were used for floral-dip transformation.

\section{Cloning and sequence analysis of $\mathrm{CtCHI}$}

The full-length cDNA sequence of $\mathrm{CtCHI}$ from Carthamus tinctorius was obtained by using $5^{\prime}$ and $3^{\prime} \mathrm{RACE}$ PCR method following the initial denaturation at $94{ }^{\circ} \mathrm{C}$ for $6 \mathrm{~min} ; 30$ cycles of $94{ }^{\circ} \mathrm{C}$ for $45 \mathrm{~s}, 58{ }^{\circ} \mathrm{C}$ for $45 \mathrm{~s}$, and $72{ }^{\circ} \mathrm{C}$ for $90 \mathrm{~s}$; and a final extension step at $72{ }^{\circ} \mathrm{C}$ for $10 \mathrm{~min}$. The appropriate pair of primer CtChir (AAT AGGATCCCGGAAGTGCAATTACCAT) and CtChif (AAT AGA ATTCCTCGTGAA ACTCCTGTT T TC 
T) containing restriction enzyme sites for EcoRI and BamHI was designed using Premier 5.0 (Additional file 1: Table S1). The amplified PCR fragments were cloned into pEASY-T1 vector (Takara, Beijing) and then sequenced to investigate any base mutation. After confirmation of Sanger sequencing, the sequence assembly of $\mathrm{CtCHI}$ was performed, and the expected amino acid sequence was deduced using DNAMAN software (Lynnon Corp., Quebec, Canada) with default parameters. The maximum open reading frame was predicted by the Laser gene SeqMan II Module (http://www.DNAstar.com). The homologous sequences were aligned by BLASTX (with $E$ value $<10^{-10}$ ) and compared against the National Center for Biotechnology Information (NCBI) database. The physicochemical characteristics of the $\mathrm{CtCHI}$ protein, including the amino acid sequence, relative molecular weight, and isoelectric point, were characterized using ProtParam software (http://web.expasy.org/). Homology analysis was carried out using DNAMAN software according to (Tamura et al. 2011).

\section{Determination of safflower yellow pigments at different flowering stages}

Four different safflower varieties were selected at different flowering stages, including bud, initial, full, and fade stages. The flower petals were collected and dried in a thermostatic drier at $55{ }^{\circ} \mathrm{C}$ for $24 \mathrm{~h}$. Each sample of the flower tissue was individually ground to a fine powder. The weight of $1 \mathrm{~g}$ powdered flower tissue was measured, followed by soaking in $25 \mathrm{ml}$ of $50 \%$ methanol solution. The resultant solution was subjected to ultrasonic extraction using the following conditions: extraction temperature, $42{ }^{\circ} \mathrm{C}$; extraction period, 30 min twice; centrifugation at $5000 \mathrm{rpm}$ for $10 \mathrm{~min}$. The supernatant was cautiously diluted in methanol and then infiltrated with a filter membrane $(0.45-\mu \mathrm{m})$. The samples were then measured by comparing the absorbance values at $300 \mathrm{~nm}$. Each experiment was performed in three independent biological replicates presented as means $\pm \mathrm{SE}$ $(\mathrm{n}=3)$. Asterisks indicate the statistical significance levels according to Student's $t$-test: ${ }^{*} \mathrm{P}<0.05$.

\section{The interrelationship between $\mathrm{CtCHI}$ expression level and safflower yellow pigment accumulation during different flowering stages}

In order to determine the correlation between $\mathrm{CtCHI}$ expression and accumulation of safflower yellow pigment, the expression level of $\mathrm{CtCHI}$ at four different flowering stages of Honghua Jihong No. 1 (JH1) cultivar was further investigated. The flower petals of the bud, initial, full, and fade stages were collected from $\mathrm{JH} 1$ cultivar and then subjected to RNA extraction using RNA isoplus. The first strand cDNA templates were prepared by reverse transcription PCR. The expression of $\mathrm{CtCHI}$ gene was calculated by real time-PCR (qRT-PCR) analysis using primer pair RTCHI-F/R (Additional file 1: Table S1). qRT-PCR reactions were measured on (Applied Biosystems 7300/7500/7500 Fast Real-Time PCR System; Foster City, CA, USA). Each qPCR reaction was performed in a total volume of $20 \mu \mathrm{l}$, containing $10 \mu \mathrm{l}$ SYBR Premix Ex Taq (Tli RNaseH Plus) $(2 \times), 0.4 \mu \mathrm{l}$ ROX Reference Dye II $(50 \times), 0.4 \mu \mathrm{l}$ of each of upstream and downstream primers $(0.2 \mu \mathrm{M}), 2 \mu \mathrm{l}$ cDNA template $(100 \mathrm{ng})$ and $6.8 \mu \mathrm{l}$ $\mathrm{ddH}_{2} \mathrm{O}$ (Bio-Engineering Co., Ltd., Jilin, China. Item No. RR420A). The qRT-PCR thermal cycle was followed as initial denaturation $\left(30 \mathrm{~s}\right.$ at $\left.95^{\circ} \mathrm{C}\right)$, followed by 40 cycles of $30 \mathrm{~s}$ at $95{ }^{\circ} \mathrm{C}$ and $3 \mathrm{~s}$ at $65^{\circ} \mathrm{C}$. All reactions were carried out in 3 independent replicates, and the results were calculated according to $2^{-\Delta \Delta \mathrm{Ct}}$ whereas $18 \mathrm{~s}$ ribosomal RNA gene (GenBank accession: AY703484.1) was used as a housekeeping gene.

\section{Subcellular localization of $\mathrm{CtCHI}$}

The subcellular localization of $\mathrm{CtCHI}$ was computationally predicted with the help of WoLF PSORT program (Horton et al. 2007). Subsequently, the $\mathrm{CtCHI}$ gene was amplified using the forward primer SLCHI-F (AAA CTAGTATGGCTCCGCCGCCGTCCAC) and reverse primer SLCHI-R (AAAGATCTATTCATGAGATCG GCCAATC) containing restriction enzyme sites for speI and $b g l I I$. The amplified fragment of $\mathrm{CtCHI}$ gene was recloned into the linearized pCAMBIA1302 vector, which consists of a green fluorescent protein (GFP) under the control of the CaMV $35 \mathrm{~S}$ promoter. After confirmation by sequencing, the recombinant product was transferred into A. tumefaciens (EHA105) via freeze and Thaw method. In the meanwhile, an empty vector of pCAMBIA1302 was also transformed into A. tumefaciens. Wild type tobacco plants were grown in a growth chamber $\left(25^{\circ} \mathrm{C}, 16 \mathrm{~h}\right.$ light $/ 8 \mathrm{~h}$ dark). Plants with at least two pairs of leaves were selected for infiltration. Prior to transient expression, the positive strains of Agrobacterium were selected and incubated in Luria-Bertani (LB) media containing $50 \mathrm{mg} / \mathrm{l} \mathrm{kanamycin}$ and $50 \mathrm{mg} / \mathrm{l} \mathrm{rifampicin}$. The transformation was performed by injecting the harvested culture of Agrobacterium at an $\mathrm{OD}_{600}$ equal to 1.0 with the help of a syringe into the leaves of healthy wild tobacco plants. The GFP fluorescence of $\mathrm{CtCHI}$ protein and the pCAMBIA1302 empty vector were visualized using a laser confocal microscopy.

\section{Vector construction and Agrobacterium-mediated transformation in Arabidopsis}

The full-length cDNA of $\mathrm{CtCHI}$ was ligated into a linearized plant over-expression vector (pBASTA). Before ligation, pEASYT1-CtCHI and empty vector of pBASTA 
was treated with double digestion system using BamHI and $E c o$ RI restriction enzymes. The digestion system was used as follow: Total volume of $20 \mu \mathrm{l}$ reaction, containing $2 \mu \mathrm{g}$ DNA, $2 \mu \mathrm{l}$ of EcoRI and BamHI, $2 \mu \mathrm{l}$ Buffer K and $\mathrm{ddH}_{2} \mathrm{O}$ respectively. Ligation with the plant overexpression vector and $\mathrm{CtCHI}$ was performed using T4 ligase sticky end ligation system according to (Jailani et al. 2016). The pBASTA-CHI expression vector, along with the empty vector, was further transformed into EHA105 E. coli Trans (T1) competent cells. Positive colonies were detected by colony PCR. The Agrobacterium-mediated transformation of wild-type Arabidopsis. thaliana was carried out following the floral-dip infection method, according to (Jiang et al. 2015).

\section{Expression profiling of $\mathrm{CtCHI}$ gene in transgenic system}

The transgenic Arabidopsis lines were grown in an artificial climate chamber up to a homozygous T3 generation. Transgenic plants were maintained in a controlled environment $\left(25^{\circ} \mathrm{C}, 16 \mathrm{~h}\right.$ light $/ 8 \mathrm{~h}$ dark $)$ until harvesting. The leaves of 14 homozygous T3 lines were collected for the isolation of total RNA in order to synthesize the first strand cDNA via reverse transcription for qRT-PCR analysis, following the protocols described above.

\section{In vitro enzymatic activity of $\mathrm{CtCHI}$}

The double antibody sandwich ELISA was carried out to detect the relative amount of $\mathrm{CtCHI}$ analyte and its associated in vitro enzymatic activity obtained from the original samples of T3 homozygous Arabidopsis mutants. The samples with an unknown amount of $\mathrm{CtCHI}$ antigen were collected from 14 transgenic plants. The samples were immobilized for $30 \mathrm{~min}$ at $37^{\circ} \mathrm{C}$ on a solid surface through a conjugation detection antibody (ELISA KIT, subpackage; RC9615 R\&D Systems Inc America). The unbound antibodies were removed through washing using wash buffer. After final washing, the plate was subjected to conjugation with HRP Enzyme-linked secondary antibodies for $30 \mathrm{~min}$ at $37^{\circ} \mathrm{C}$ in order to produce a visible signal. The plate was thoroughly rinsed with wash buffer to detach the unbound antibody-enzyme conjugates present in the samples and subsequently stained with a chemical 3,3'5,5'-tetramethylbenzidine (TMB) substrate to the wells resulted in blue color fluorescent indicating the amount of analyte present in the samples. The turning of blue color signals into yellow signals indicated the nonspecific reaction of HRP-labeled antibody. The development of the electrochemical signals (Blue/ Yellow) obtained from 14 mutant Arabidopsis was measured as absorbance (OD) using a spectrophotometer at $300 \mathrm{~nm}$ to determine the quantity of $\mathrm{CtCHI}$ antigen in the samples.

\section{Determination of flavonoids accumulation in transgenic Arabidopsis}

The leaves of transgenic Arabidopsis were collected and grinded, and then passed through a 40-mesh screen. Accurately weighted $(0.5 \mathrm{~g})$ of fine leaf powder was soaked in $50 \mathrm{ml}$ methanol (65\%) solution followed by sonication for $30 \mathrm{~min}$ with the following conditions: temperature $50{ }^{\circ} \mathrm{C}$, ultrasonic wavelength $600 \mathrm{~W}$ with $40 \mathrm{kHz}$ frequency. The resultant extract was carefully diluted in methanol solution and then purified through a filter membrane. After centrifugation at $12,000 \mathrm{rpm}$ for $10 \mathrm{~min}$, the supernatant was collected, filtered, and analyzed by HPLC analysis following the protocol of (Sun et al. 2016). The separation of Chromatic compounds was performed using different gradient solutions of the authentic standard of Rutin. Six different gradient solutions were prepared by dissolving $2,4,6,8,10$, and $12 \mathrm{ml}$ Rutin in $20 \mathrm{ml}$ methanol. Chromatographic conditions includes: Column: Agilent zorbax SB-C 18 (4.6 $\mathrm{mm} \times 150 \mathrm{~mm}, 5 \mu \mathrm{m})$; mobile phase: phase $A$ is methanol-acetonitrile (V: V 1:10), phase B is $0.4 \%$ phosphoric acid solution. The gradient elution went from [ 94 . $5 \% \mathrm{~B} \rightarrow 86.8 \% \mathrm{~B}$ in $10 \mathrm{~min}, 20 \mathrm{~min}, 86.8 \% \mathrm{~B} \rightarrow 81.3 \% \mathrm{~B}$ in $20 \mathrm{~min}, 81.3 \% \mathrm{~B} \rightarrow 80.75 \% \mathrm{~B}$ in $40 \mathrm{~min}, 80.75 \% \mathrm{~B} \rightarrow 76$. $9 \% \mathrm{~B}$ in $40 \mathrm{~min}, 76.9 \% \mathrm{~B} \rightarrow 70.3 \% \mathrm{~B}$ in $50 \mathrm{~min}]$. Measured the detection wavelength at $340 \mathrm{~nm}$; Column temperature: $25^{\circ} \mathrm{C}$; at a Flow rate of $0.8 \mathrm{ml} / \mathrm{min}$ and Volume: $20 \mu \mathrm{l}$ with three replicates.

\section{Results \\ Cloning, sequence analysis and characterization of the $\mathrm{CtCHI}$ gene}

We previously analyzed the high throughput transcriptomic analysis obtained from RNA sequencing of safflower petals in order to discover the Safflower yellow pigment biosynthetic related genes (Liu et al. 2015). In the current study, the full-length cDNA (1161 bp) of the CtCHI gene (GenBank accession: KP300882) was cloned using $5^{\prime}$ and $3^{\prime}$ rapid amplification (Fig. 2a). Sequence analysis showed that $C t C H I$ contained an open reading frame of $654 \mathrm{bp}$ encoding a polypeptide with 217 amino acids. It was found that the length of $5^{\prime}$ untranslated region ( $5^{\prime}$ UTR) consist of $250 \mathrm{bp}$ while the $3^{\prime}$ untranslated region ( $3^{\prime}$ UTR) was found 257 bp including a typical Poly (A) tail. Three-dimensional protein structure prediction confirmed that the protein encoded by $\mathrm{CtCHI}$ gene was a monomer with $23.14 \mathrm{Ku}$ of theoretical molecular weight and had an isoelectric point (pI) of 5.67 which is somewhat homologous $(53.52 \%)$ to the template proteins in the SWISS-MODEL database (https ://swissmodel.expasy.org/repository) (Fig. 2b). BLASTN query from the NCBI database confirmed the grouping 


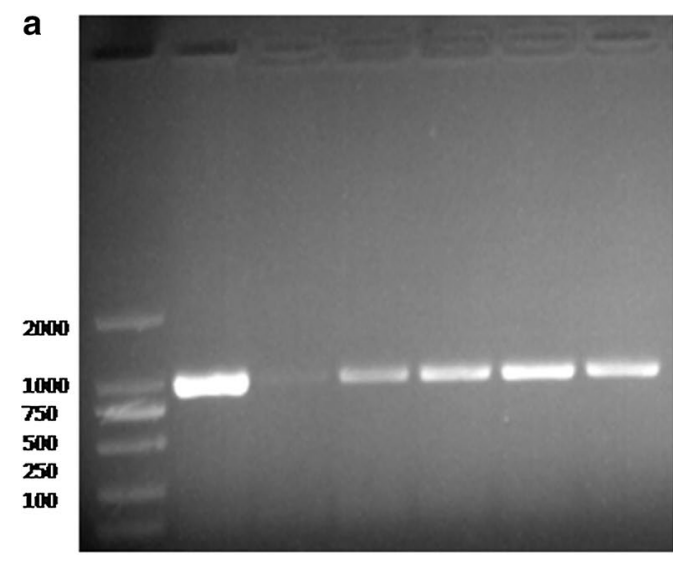

C

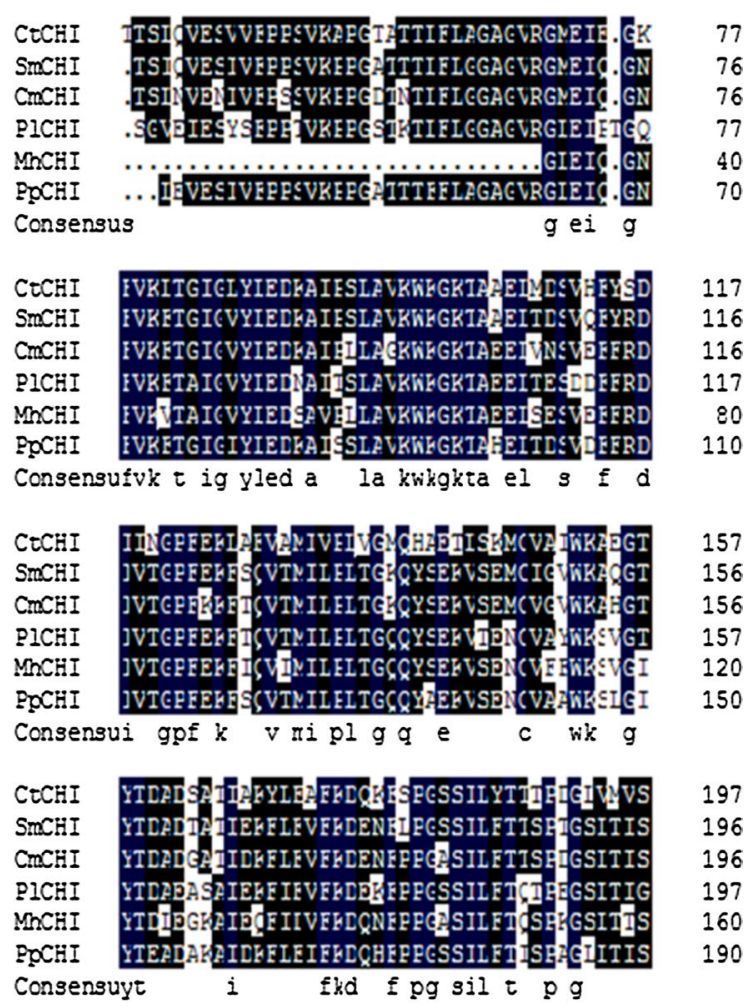

Fig. 2 Cloning and sequence analysis of the CtCHI gene. a cDNA of CtCHI. b Tertiary 3D structure of the CtCHI protein. $\mathbf{c}$ Alignment of the deduced amino acid sequence of CtCHI gene with other plant species. CtCHI: Carthamus tinctorius L.; SmCHI: Saussurea medusa; pl CHI: Paeonia lactiflora; Mh $\mathrm{CHI}$ : Malus hybrid; Pp CHI: Pyrus pyrifolia

of $\mathrm{CtCHI}$ to chalcone superfamily. Further phylogenetic analysis revealed the comparative homology of the $\mathrm{CtCHI}$ gene with other CHIs from other five dissimilar species including dahlia (65.97\%), chrysanthemum (66.17\%), apple (50.99\%), Aristolochia debilis (69.17) and peony (69.55\%). Based on the phylogenetic divergence exhibited by $\mathrm{CtCHI}$ gene in these uncommon species confirms the hypothetic role of a new Chalcone isomerase in Carthamus tinctorius (Fig. 2c).

\section{Measurement of safflower yellow pigment at various flowering periods}

The yellow pigment of safflower was extracted from four different varieties of Carthamus tinctorius including (JHEM, JHI, JHS, and JH2). The contents of Safflower yellow pigment at four different flowering stages consisting of bud, initial, full, and fade stage were determined using spectrophotometric measurement, respectively (Fig. 3). A unique pattern of the ultra-violet wavelength at $300 \mathrm{~nm}$ was strikingly absorbed for the yellow pigment accumulation during all four varieties. However, the amount of yellow pigment during the full flowering stage was found significantly higher than the other three stages in each variety. Out of the four varieties, JH1 had the highest amount of yellow pigments synthesis occurred during the full stage, reaching $3.791 \mathrm{mg} / \mathrm{ml}$. Therefore, JH1 was used as the experimental object in our study.

\section{Correlation between $\mathrm{CtCHI}$ transcript abundance and SYA accumulation in safflower}

After the selection of JH1 safflower cultivar displaying the maximum accumulation of safflower yellow pigment, we further investigated the correlation between the transcript abundance of $\mathrm{CtCH} 1$ gene and safflower yellow pigment accumulation that took place at different flowering stages using qRT-PCR analysis. It was observed that the expression level of $\mathrm{CtCHI}$ gene at bud stage was found greater than the other three stages. However, the yellow pigment accumulation was consistently remained higher at the full stage with the notably decreased expression level of CtCHI than in bud, initial, and fade stages (Fig. 4). Moreover, the accumulation pattern of safflower yellow pigment at $\mathrm{CHI}$-Initial and $\mathrm{CHI}$-fading stages were appeared to synthesize a 


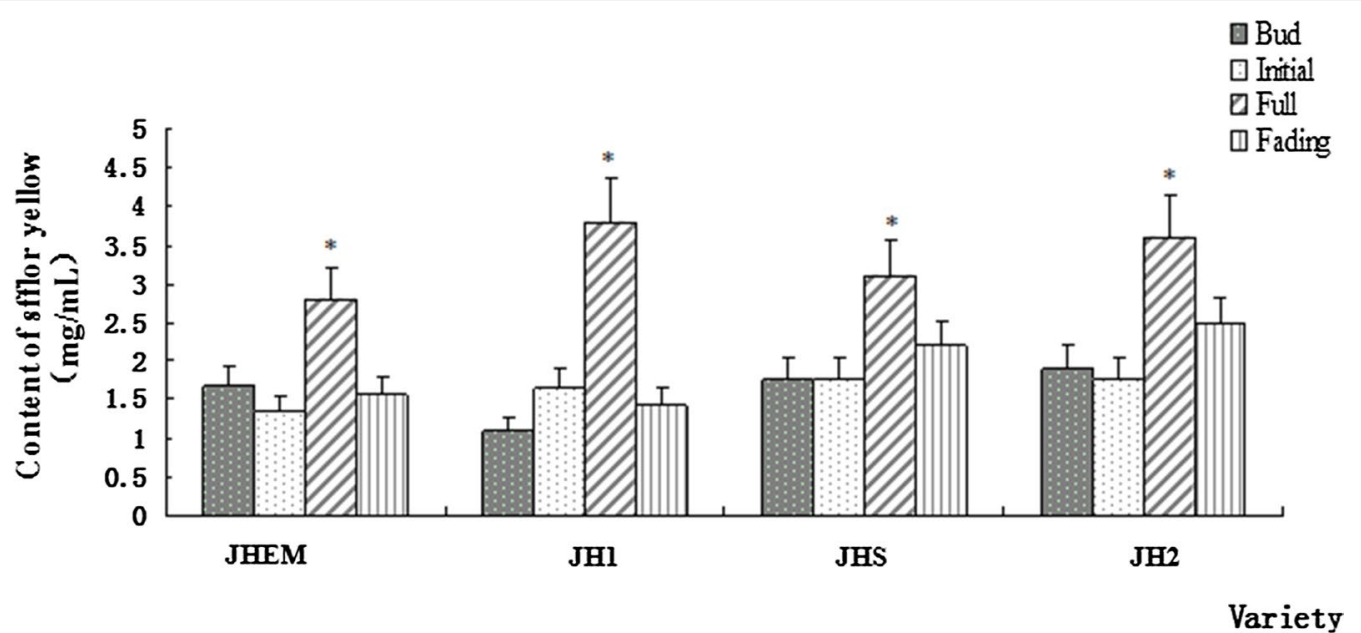

Fig. 3 The content of yellow pigment of safflower accumulated during four different flowering periods in four different varieties. Safflower varieties were labeled as JHEM, JH1, JHS, and JH2. Different flowering periods were indicated as a bud, initial, full, and fading stage. Data are means \pm SE $(n=3)$. Asterisks indicate the statistical significance levels according to Student's $t$-test: ${ }^{*} P<0.05$

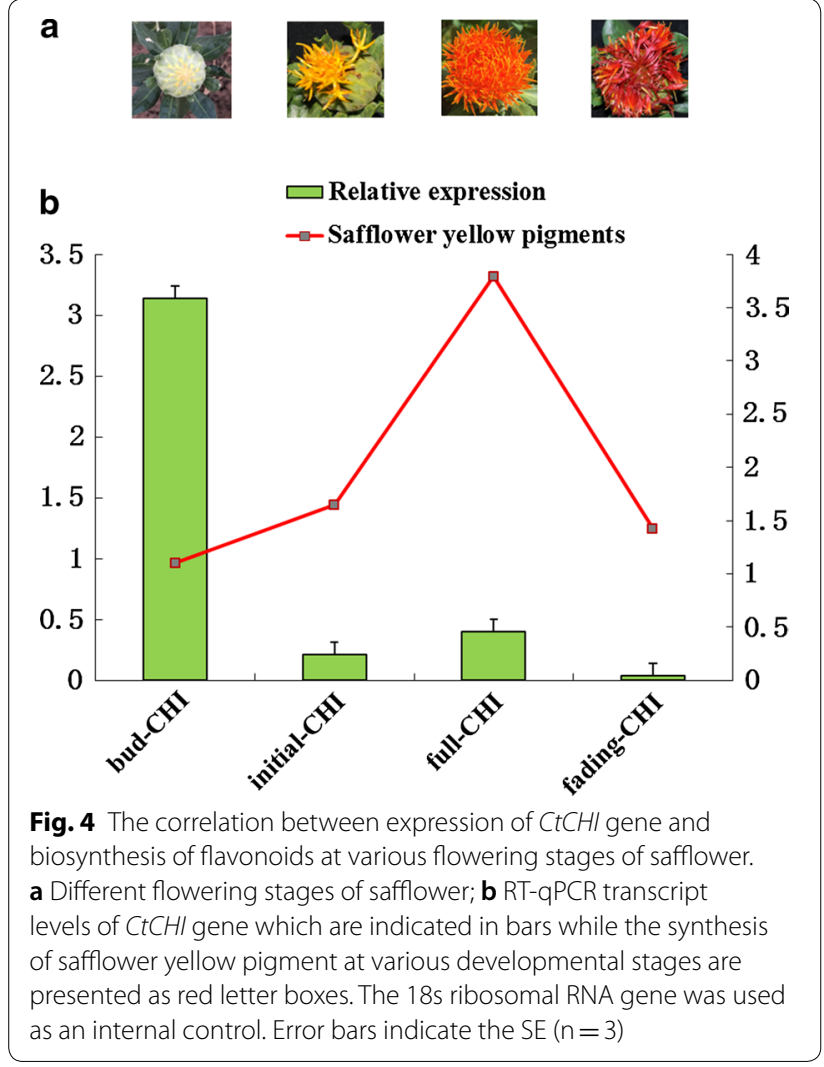

relatively similar amount of yellow pigment. Nevertheless, the expression pattern of $\mathrm{CtCHI}$ showed a reverse order, suggesting the metabolon mechanism of $\mathrm{CtCHI}$ during both of these stages occurred from the similar upstream gene, or it may be the result of a synergistic consequence on safflower yellow pigment metabolism at this specific junction. Furthermore, the accumulation of a moderate level of yellow pigment at $\mathrm{CHI}$-bud stage with the relatively increased expression level of $\mathrm{CtCHI}$ at this stage indicated the importance of $\mathrm{CtCHI}$ gene during flavonoid synthesis in Carthamus tinctorius.

\section{Subcellular localization of $\mathrm{CtCH}$ using GFP tagging}

The theoretical prediction of $\mathrm{CtCHI}$ protein revealed a $60.4 \%$ probability to be located in the nucleus and $17.5 \%$ of probability localization in the cytoplasm. Then, we investigated the precise subcellular localization of CtCHI gene through Green fluorescent protein (GFP) tagging using a fusion construct of pCAMBIA1302 vector and $\mathrm{CtCHI}$ under the control of CaMV $35 \mathrm{~S}$ promoter. In this report, the pCAMBIA1302 empty vector was used as a control. Wild type tobaccos were infected with pCAMBIA1302-CtCHI and PCAMBIA1302 alone via Agrobacterium (EHA105) mediation. The GFP fluorescence of $C t C H I$ gene and the empty vector was analyzed under a confocal laser scanning microscope. As described in Fig. 5, the tobacco plants harboring the CtCHI-pBASTA1302-GFP vector exhibited GFP signals predominately in nucleus and cytoplasm. On the contrary, tobacco carrying pBASTA1302-GFP alone was detected solely inside mesophyll cells. These findings indicated that $\mathrm{CtCHI}$ was originally localized in the nucleus and therefore could possibly regulate a physiological pathway in Carthamus tinctorius. 


Bright
CtCHI
pCAMBIA1302
Fig. $\mathbf{5}$ Subcellular localization of CtCHI-GFP in wild type tobacco mesophyll cells. GFP signals of CtCHI-pBASTA1302-GFP fusion construct localizes
to both cytosol and nucleus. The pBASTA1302-GFP signals were detected dispersed in tobacco mesophyll cells. The GFP fluorescence exhibited
signals were analyzed with a confocal laser scanning microscope

\section{Identification of $\mathrm{CtCH}$ overexpressed T3 Arabidopsis}

Transgenic seeds were grown up to T3 generation, and then selection and screening of the homozygous T3 transgenic plants were performed by spraying $1 \%$ Basta (Additional file 1: Fig. S3). The use of antibiotic selection could eliminate several drawbacks when generating and identifying transgenic plants simply by removing fungal contamination. It can also help to screen out those lines which were internally contaminated by the Agrobacterium strain itself. Further identification of the mutant plants was carried out using the $\mathrm{BAR}$ gene as a selectable marker to detect the presence of transgene pBASTA-CtCHI (Additional file 1: Fig. S1). Additionally, we examined the presence of terminator gene (NOS) in the selected transgenic lines of Arabidopsis (Additional file 1: Fig. S2). For this purpose, the genomic DNA of transgenic plants was extracted with the help of NuClean plant Genomic DNA Kit (Beijing, China). PCR amplification of CtCHI, BAR gene, and NOS terminator gene was performed respectively using $G^{\circ}$ Taq DNA polymerase (Promega Corp, Madison, WI) with the help of the respective pair of primers (Additional file 1: Table S1).

\section{Quantitative real-time expression and in vitro activity} of $\mathrm{CtCH}$ in transgenic Arabidopsis

The leaves of 14 homozygous Arabidopsis T3 lines and WT plants of Arabidopsis were collected and subject to RNA extraction for synthesizing the template cDNA through reverse transcription PCR. The expression level of the CtCHI gene in the transgenic Arabidopsis lines was normalized using $18 \mathrm{~S}$ ribosomal RNA as an internal reference gene in the qRT-PCR system. According to Fig. 6, the expression analysis of 14 transgenic lines showed a correspondently higher level of $C t C H I$ mRNA transcript abundance accumulated in the AR-CHI-9 followed by AR-CHI-1 mutant line in comparison to other transgenic and wild-type plant. According to the core metabolic biosynthetic pathway in safflower, the expression of $\mathrm{CtCHI}$ should be negatively correlated with metabolite biosynthesis, however, the opposed pattern of CtCHI mRNA transcripts in AR-CHI-9 and $A R-C H I-1$ transgenic systems implied the potential role of $\mathrm{CtCHI}$ during metabolite biosynthesis.

Furthermore, to investigate the interrelationship of $\mathrm{CtCHI}$ expression level and $\mathrm{CtCHI}$ in vitro enzymatic activity, we measured the relative mass values of the 


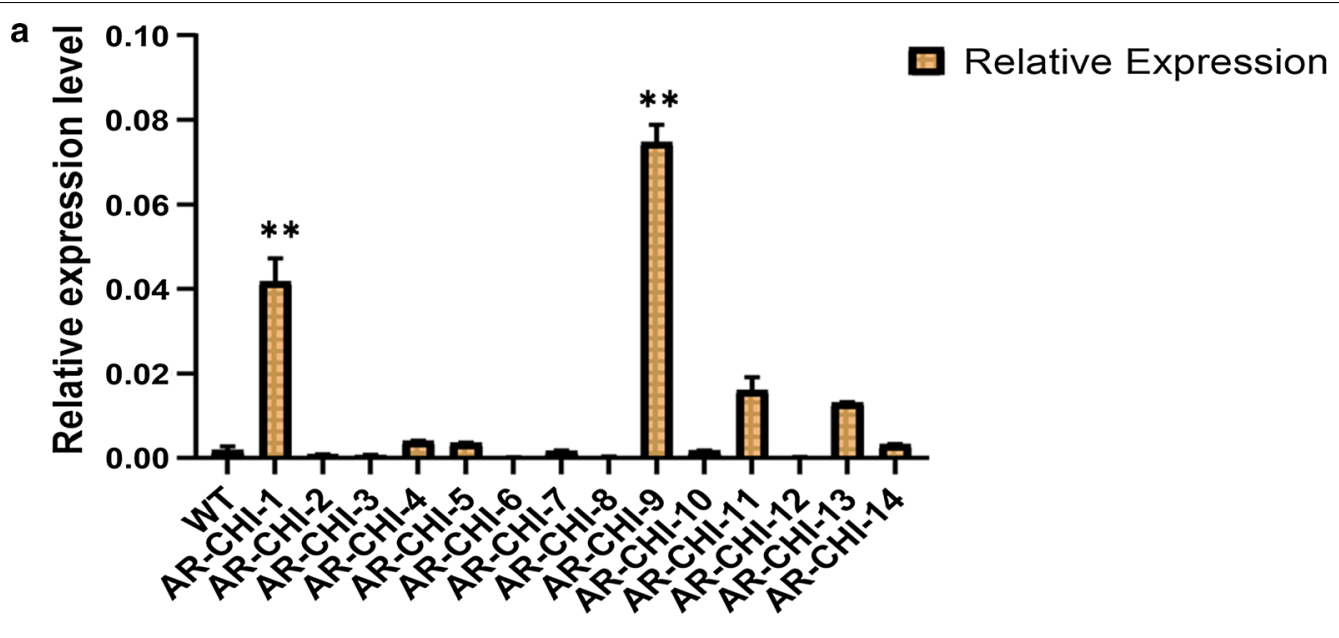

b

Transgenic plants

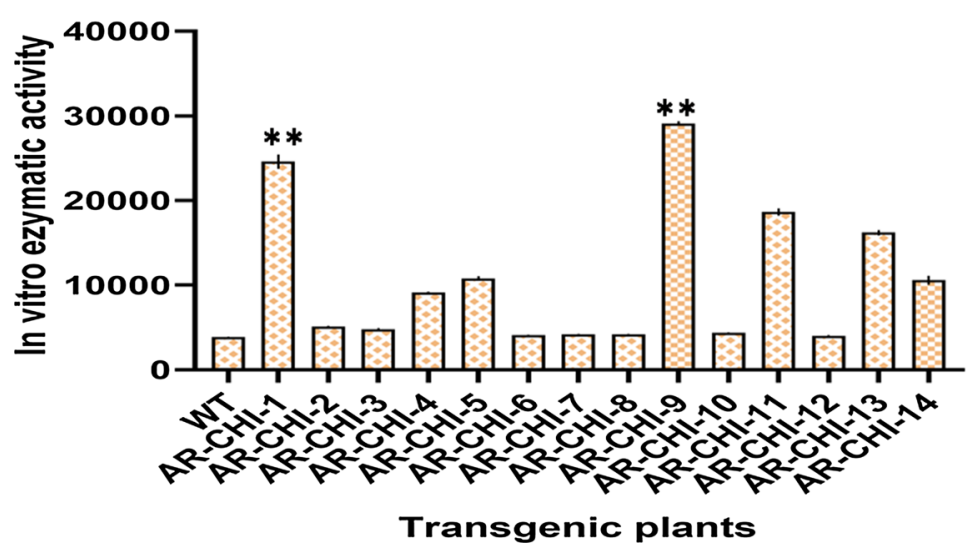

-In vitro Activity

Transgenic plants

Fig. 6 Quantitative real time Expression levels of $\mathrm{CtCH}$ gene and $\mathrm{CH}$ activity in transgenic Arabidopsis lines. a Relative expression levels of $\mathrm{CtCHI}$ gene presented in four different transgenic lines in comparison with the wild type Arabidopsis. The data were normalized with 18s ribosomal RNA. b In vitro enzymatic activity of CHI using ELISA kit, RC9615, R\&D Systems Inc., America). The color of the 3,3',5,5'-tetramethylbenzidine was measured as absorbance (OD) using a spectrophotometer at $450 \mathrm{~nm}$ to calculate the concentration of $\mathrm{CH}$ activity according to a standard regression curve. Data were presented as the mean $\pm S D$. ${ }^{* *} \mathrm{P}<0.01$ vs. the wild type group

natural analyte present in the selected homozygous T3 lines using double antibody sandwich ELISA method (see "Materials and methods" for details). The potential of $R \& D$ Systems to determine the supreme standard curve range for individual sample by asserting results of peak sensitivity and reproducibility. Our findings indicated the increased $C t C H I$ activities in two transgenic Arabidopsis lines (AR-CHI-9 and AR-CHI-1), which exhibited the highest electrochemical signals resulted in blue color visualization at absorbance (OD) equals to $450 \mathrm{~nm}$. It was found noteworthy that the expression level of $\mathrm{CtCHI}$ transcript abundance in the transgenic systems of $A R$ CHI-9 and AR-CHI-1 were found consistent and with a parallel manner to their in vitro enzymatic activities, as shown in Fig. 6. However, the lower $\mathrm{CHI}$ expression in other transgenic plants was not likely associated with in vitro activity of $\mathrm{CtCHI}$, and likely have to do with procyanidin or other types of pigments present early maturation phases. Based on these findings, we speculate the partial function of $\mathrm{CtCHI}$ providing adequate insights during flavonoid biosynthesis.

\section{HPLC analysis in transgenic Arabidopsis}

Wild-type Arabidopsis plants normally display a mixture of quercetin and kaempferol glycosides. The standard peaks of these compounds were identified (data not shown) and compared with solvent extract peaks obtained from transgenic Arabidopsis T3 plants expressing the putative $\mathrm{CHI}$ gene. According to the standard curve of linear regression, the accumulation of increased 


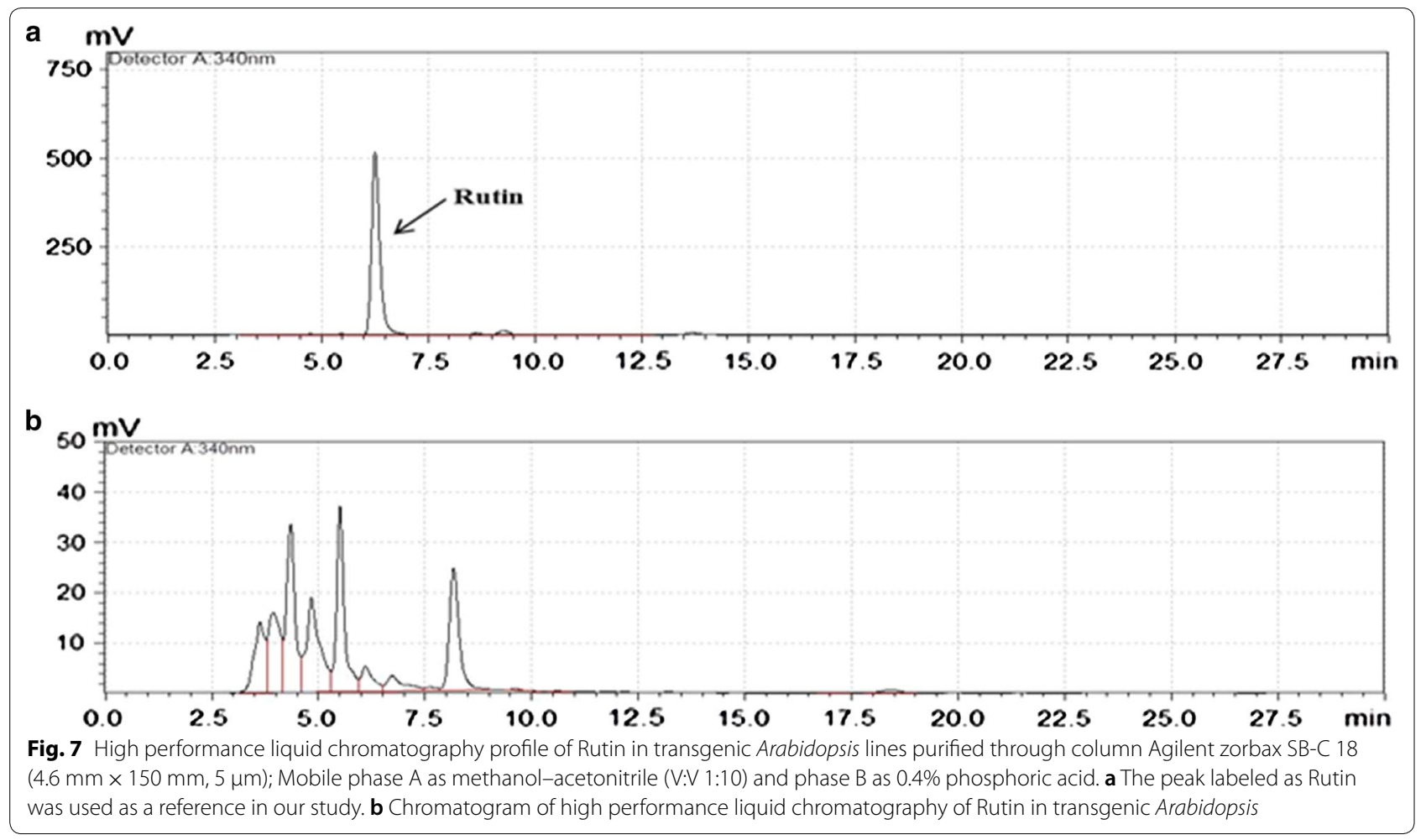

quercetin-glycoside (rutin) reaching up to $(187.37 \mu \mathrm{g} /$ $\mathrm{mg}$ ) in transgenic $A R-C H I-9$ line followed by $A R-C H I-1$ $(173.67 \mu \mathrm{g} / \mathrm{mg})$ is noteworthy as shown in the HPLC chromatogram (Fig. 7). The increased tendency of Rutin accumulation in $A R-C H I-9$ and $A R-C H I-1$ showed a similar pattern in comparison to $\mathrm{CtCHI}$ relative expression and in vitro $\mathrm{CHI}$ activity in these lines. Altogether, these results highlight the identification of a new $\mathrm{CtCHI}$ from Carthamus tinctorius promoting flavonoid biosynthesis.

\section{Discussions}

Chalcone isomerase is one of several important enzymes in the biosynthesis of flavonoids, catalyzing the stereospecific isomerization of chalcones into their corresponding (-)-flavanones catalysis (Cheng et al. 2011). Several studies have demonstrated the roles of the CHI gene in the regulation of plant metabolism and changes in Flavonoid components in flower petals (Nishihara et al. 2005). However, the explicit expression pattern of $\mathrm{CHI}$ gene in flavonoid metabolic pathway in safflower remains unclear. In this study, the full-length cDNA of $\mathrm{CtCHI}$ gene was cloned from safflower petals using reverse transcription polymerase chain reaction. Blast result and phylogenetic analysis revealed that the $\mathrm{CHI}$ gene is highly homologous to $\mathrm{CHI}$ gene of the other plant species, indicating that this gene is highly conserved in plants which were consistent with the results of (Hong et al. 2012). The biosynthetic class of enzymes resides majorly in the nucleus where they play an essential role as DNA protectants against Ultra-Violet radiation as well as they are capable of regulating other related genes in one way or another which are necessary for other important physiological processes (Polster et al. 2006). Subcellular localization analysis localized the $\mathrm{CtCHI}$ gene on the cell membrane and in the nucleus, disagreeing with the previous results of (Dastmalchi and Dhaubhadel 2015), who reported the $\mathrm{CHI}$ gene on the nucleus and in the cytoplasm of soybean. Differences of the chromosomal locations of this gene might vary among plant species (Lv et al. 2017).

The in vitro enzymatic activity of $\mathrm{CtCHI}$ in $\mathrm{AR}-\mathrm{CHI}-1$ and AR-CHI-9 transgenic Arabidopsis lines exhibited the highest activity in catalyzing the synthesis of Rutin than all mutant plants and wild type plants. Additionally, the expression pattern of $\mathrm{CtCHI}$ in these plants was also consistent with it's in vitro activity, which implies the positive role of $\mathrm{CtCHI}$ in flavonoids metabolism in safflower. Chalcone isomerase gene has been proven necessary for the biosynthesis of flavonoids and other essential pigments (Morita et al. 2014). We, therefore, analyze the expression levels of the $\mathrm{CtCHI}$ gene which indicates significant differences at various stages of flower development in safflower, peaking at the bud stage when the amount of pigments is the lowest and 
declining at the full stage when the content of pigments was at peaks. The result provides considerable evidence for the partial function of $\mathrm{CHI}$ gene during the regulation of floral pigmentation but the expression levels of CtCHI didn't significantly correlate with pigments content, speculating that at the bud stage the flower color undergoes from faint yellow to yellow, while the expression of the CHI was relatively low in the more stable full stage.

The CHI gene is the main structural gene encoding the most important rate-limiting enzyme in the biosynthetic pathway of flavonoids. We also identified other putative CHIs in safflower (Additional file 1: Fig. S4) however, the expression level of these putative CHIs showed relatively lower expression level in comparison to $\mathrm{CtCHI}$ at the bud stage of JH1 cultivar. Previous studies have shown that the expression of the $\mathrm{CHI}$ gene in plants greatly influence flavonoid biosynthesis (Jiang et al. 2015; Nishihara et al. 2005; Wang et al. 2015). In many plant species, mutations with a deletion of the $\mathrm{CHI}$ gene may limit some steps in flavonoid biosynthesis, leading to a significant decrease in the content of flavonoids and anthocyanins produced (Kim et al. 2004; Van Tunen et al. 1988). In the present study, the certain increase of the amounts of flavonoids was detected in two transgenic Arabidopsis lines, consistent with previous studies (Muir et al. 2001; Park et al. 2011; Zhang et al. 2009). The result of this study provides supportive evidence for an active relationship between $\mathrm{CHI}$ gene expression and flavonoid synthesis. By cloning a CHI gene from Saussurea medusa and thereby over-expressing it in transgenic tobacco, (Li et al. 2006) reported that transgenic plants produced up to fivefold more total flavonoids over WT plants. Overexpression of the $\mathrm{CHI}$ gene in transgenic plants is an effective method for enhancing the content of flavonoids. In our findings, however, the number of flavonoids produced in transgenic Arabidopsis lines was maximally increased by twofold over WT plants. This has occurred because $\mathrm{CHI}$ is a rate-limiting enzyme (Muir et al. 2001) at the upstream of the flavonoid biosynthetic pathway, during which many important enzymes are involved in the regulation of the complex process of flavonoid biosynthesis. Over-expression of $\mathrm{CHI}$ may stimulate the expression of other key enzymes to facilitate flavonoid metabolism. In this study, $A R-C H I-9$ had the highest yield of flavonoids than other transgenic Arabidopsis mutants. This might occur as a result of differential gene expression pattern influenced by different environmental factors or other internal reasons; this could be further verified by phenotypic experiments in future researches.

\section{Additional file}

Additional file 1: Table S1. List of primers. Fig. S1. Plant over expression vector. Fig. S2. Detection of transgenic plants. Fig. S3. Strongest transgenic Arabidopsis lines obtained in our study. Fig. S4. Other putative CHls in safflower. Fig. S5. Graphical representation of pCAMBIA1302-GFP$35 \mathrm{~S}$ vector.

\section{Abbreviations}

GFP: green fluorescent protein; HPLC: high performance liquid chromatography; Pl: isoelectric points; $\mathrm{kDa}$ : kilo dalton; CDS: coding sequences; qRT-PCR: quantitative real-time PCR; MW: molecular weight; JH1: Jihong No. 1 (safflower cultivar); WT: wild-type; CaMV: cauliflower mosaic virus; LB: Luria-Bertani; ELISA: enzyme-linked immunosorbent assay.

\section{Acknowledgements}

We thank Mallory Eckstut, PhD, from Liwen Bianji, Edanz Editing China (http:// www.liwenbianji.cn/ac), for editing the English language of this manuscript.

\section{Authors' contributions}

Research design, Xiuming Liu; Data analysis, TF and FW; Formal analysis, LY, NY, NW, and Xiaowei Li; Funding acquisition, HL; Investigation, Xin Liu and YD; Methodology, NA; Software, JK; Writing-original draft, Xiuming Liu; Writingreview and editing, NA and WL. All authors read and approved the final manuscript.

\section{Funding}

This work was funded by grants from the National High Technology Research and Development Program of China (863 Program) (No. 2011AA100606), the National Natural Science Foundation of China (Nos. 31771868, 31501366), the Science and Technology Development Project of Jilin Province (Nos. 20150623024TC-11, 20170520089JH).

\section{Availability of data and materials}

All the data which is generated and analyzed during our study are included in the article and additional files.

\section{Ethics approval and consent to participate}

Not applicable.

\section{Consent for publication}

Not applicable.

\section{Competing interests}

The authors declare that they have no competing interests.

\section{Author details}

${ }^{1}$ Ministry of Education Engineering Research Center of Bioreactor and Pharmaceutical Development, Jilin Agricultural University, Changchun 130118,

China. ${ }^{2}$ College of Life Sciences, Jilin Agricultural University, Changchun 130118, China.

Received: 1 April 2019 Accepted: 8 August 2019

Published online: 21 August 2019

\section{References}

Cheng H, Li L, Cheng S, Cao F, Wang Y, Yuan H (2011) Molecular cloning and function assay of a chalcone isomerase gene (GbCHI) from Ginkgo biloba. Plant Cell Rep 30(1):49-62

Dai Y, Witkamp G-J, Verpoorte R, Choi YH (2013) Natural deep eutectic solvents as a new extraction media for phenolic metabolites in Carthamus tinctorius L. Anal Chem 85(13):6272-6278

Dastmalchi M, Dhaubhadel S (2015) Soybean chalcone isomerase: evolution of the fold, and the differential expression and localization of the gene family. Planta 241(2):507-523

Gensheimer M, Mushegian A (2004) Chalcone isomerase family and fold: no longer unique to plants. Protein Sci 13(2):540-544 
Guo D-D, Liu F, Tu Y-H, He B-X, Gao Y, Guo M-L (2016) Expression patterns of three UGT genes in different chemotype safflower lines and under MeJA stimulus revealed their potential role in flavonoid biosynthesis. PLOS ONE 11(7):e0158159

Guo D, Xue Y, Li D, He B, Jia X, Dong X, Guo M (2017) Overexpression of CtCHS1 increases accumulation of quinochalcone in safflower. Front Plant Sci 8:1409

Gutierrez E, García-Villaraco A, Lucas JA, Gradillas A, Gutierrez-Mañero FJ, Ramos-Solano B (2017) Transcriptomics, targeted metabolomics and gene expression of blackberry leaves and fruits indicate flavonoid metabolic flux from leaf to red fruit. Fron Plant Sci 8:472

Hong L, Qian Q, Tang D, Wang K, Li M, Cheng Z (2012) A mutation in the rice chalcone isomerase gene causes the golden hull and internode 1 phenotype. Planta 236(1):141-151

Horton P, Park K-J, Obayashi T, Fujita N, Harada H, Adams-Collier C, Nakai K (2007) WoLF PSORT: protein localization predictor. Nucleic acids Res 35(2):W585-W587

Jailani AAK, Kumar A, Mandal B, Sivasudha T, Roy A (2016) Agroinfection of tobacco by croton yellow vein mosaic virus and designing of a replicon vector for expression of foreign gene in plant. Virusdisease 27(3):277-286

Jamalan M, Ghaffari MA, Hoseinzadeh P, Hashemitabar M, Zeinali M (2016) Human sperm quality and metal toxicants: protective effects of some flavonoids on male reproductive function. Int J Fertil Steril 10(2):215

Jiang W, Yin Q, Wu R, Zheng G, Liu J, Dixon RA, Pang Y (2015) Role of a chalcone isomerase-like protein in flavonoid biosynthesis in Arabidopsis thaliana. J Exp Bot 66(22):7165-7179

Kang J-H, McRoberts J, Shi F, Moreno J, Jones D, Howe GA (2014) The flavonoid biosynthetic enzyme chalcone isomerase modulates terpenoid production in glandular trichomes of tomato. Plant Physiol 164:1161-1174

Kim S, Jones R, Yoo K, Pike L (2004) Gold color in onions (Allium cepa): a natural mutation of the chalcone isomerase gene resulting in a premature stop codon. Mol Genet Genom 272(4):411-419

Kranner I, Birtić S (2005) A modulating role for antioxidants in desiccation tolerance. Integr Comp Biol 45(5):734-740

Landry LG, Chapple CC, Last RL (1995) Arabidopsis mutants lacking phenolic sunscreens exhibit enhanced ultraviolet-B injury and oxidative damage. Plant Physiol 109(4):1159-1166

Li S (2014) Transcriptional control of flavonoid biosynthesis: fine-tuning of the MYB-bHLH-WD40 (MBW) complex. Plant Signal Behav 9(1):e27522

Li F, Jin Z, Qu W, Zhao D, Ma F (2006) Cloning of a cDNA encoding the Saussurea medusa chalcone isomerase and its expression in transgenic tobacco. Plant Physiol Biochem 44(7-9):455-461

Liu X, Dong Y, Yao N, Zhang Y, Wang N, Cui X, Li X, Wang Y, Wang F, Yang J (2015) De novo sequencing and analysis of the safflower transcriptome to discover putative genes associated with safflor yellow in Carthamus tinctorius L. Int J Mol Sci 16(10):25657-25677

Lv G-Y, Guo X-G, Xie L-P, Xie C-G, Zhang X-H, Yang Y, Xiao L, Tang Y-Y, Pan X-L, Guo A-G (2017) Molecular characterization, gene evolution, and expression analysis of the fructose-1, 6-bisphosphate aldolase (FBA) gene family in wheat (Triticum aestivum L.). Front Plant Sci 8:1030

Meng C, Zhang S, Deng Y-S, Wang G-D, Kong F-Y (2015) Overexpression of a tomato flavanone 3-hydroxylase-like protein gene improves chilling tolerance in tobacco. Plant Physiol Biochem 96:388-400
Morita Y, Takagi K, Fukuchi-Mizutani M, Ishiguro K, Tanaka Y, Nitasaka E, Nakayama M, Saito N, Kagami T, Hoshino A (2014) A chalcone isomerase-like protein enhances flavonoid production and flower pigmentation. Plant J 78(2):294-304

Muir SR, Collins GJ, Robinson S, Hughes S, Bovy A, De Vos CR, van Tunen AJ, Verhoeyen ME (2001) Overexpression of petunia chalcone isomerase in tomato results in fruit containing increased levels of flavonols. Nat Biotechnol 19(5):470

Nishihara M, Nakatsuka T, Yamamura S (2005) Flavonoid components and flower color change in transgenic tobacco plants by suppression of chalcone isomerase gene. FEBS Lett 579(27):6074-6078

Park NI, Xu H, Li X, Kim S-J, Park SU (2011) Enhancement of flavone levels through overexpression of chalcone isomerase in hairy root cultures of Scutellaria baicalensis. Funct Integr Genom 11(3):491-496

Polster J, Dithmar H, Burgemeister R, Friedemann G, Feucht W (2006) Flavonoids in plant nuclei: detection by laser microdissection and pressure catapulting (LMPC), in vivo staining, and uv-visible spectroscopic titration. Physiol Plant 128(1):163-174

Sun W, Liang L, Meng X, Li Y, Gao F, Liu X, Wang S, Gao X, Wang L (2016) Biochemical and molecular characterization of a flavonoid 3-O-glycosyltransferase responsible for anthocyanins and flavonols biosynthesis in Freesia hybrida. Front Plant Sci 7:410

Tamura K, Peterson D, Peterson N, Stecher G, Nei M, Kumar S (2011) MEGA5: molecular evolutionary genetics analysis using maximum likelihood, evolutionary distance, and maximum parsimony methods. Mol Biol Evol 28(10):2731-2739

Tu Y, Liu F, Guo D, Fan L, Zhu Z, Xue Y, Gao Y, Guo M (2016) Molecular characterization of flavanone 3-hydroxylase gene and flavonoid accumulation in two chemotyped safflower lines in response to methyl jasmonate stimulation. BMC Plant Biol 16(1):132

Van Tunen A, Koes R, Spelt C, Van der Krol A, Stuitje A, Mol J (1988) Cloning of the two chalcone flavanone isomerase genes from Petunia hybrida: coordinate, light-regulated and differential expression of flavonoid genes. EMBO J 7(5):1257-1263

Wang R, Zhan S, Zhao T, Zhou X, Wang C (2015) Positive selection sites in tertiary structure of Leguminosae Chalcone isomerase. Genet Mol Res 14(1):1957-1967

Wang H, Wang W, Zhan J, Yan A, Sun L, Zhang G, Wang X, Ren J, Huang W, $\mathrm{Xu} \mathrm{H}$ (2016) The accumulation and localization of chalcone synthase in grapevine (Vitis vinifera L.). Plant Physiol Biochem 106:165-176

Yaginuma S, Shiraishi T, Igarashi K (2003) Developmental transition of the flavonoid contents in safflower leaves during stress-loaded cultivation. Biosci Biotechnol Biochem 67(8):1691-1698

Zhang H-C, Liu J-M, Lu H-Y, Gao S-L (2009) Enhanced flavonoid production in hairy root cultures of Glycyrrhiza uralensis Fisch by combining the overexpression of chalcone isomerase gene with the elicitation treatment. Plant Cell Rep 28(8):1205-1213

\section{Publisher's Note}

Springer Nature remains neutral with regard to jurisdictional claims in published maps and institutional affiliations.

\section{Submit your manuscript to a SpringerOpen ${ }^{\odot}$ journal and benefit from:}

- Convenient online submission

- Rigorous peer review

- Open access: articles freely available online

- High visibility within the field

Retaining the copyright to your article

Submit your next manuscript at $\boldsymbol{\nabla}$ springeropen.com 